\title{
Dinamit Destekli Terör Faaliyetlerinin Önlenmesi İçin Derin Öğrenme Temelli Güvenlik Destek Sistemi
}

\author{
Volkan Kaya ${ }^{1 *}$, Ahmet Baran ${ }^{1}$, Servet Tuncer ${ }^{2}$ \\ ${ }^{\mathbf{1}}$ Erzincan Binali Yıldırım Üniversitesi, Mühendislik Fakültesi, Bilgisayar Mühendisliği Bölümü, Erzincan, Türkiye (ORCID: 0000-0001-6940-3260), \\ vkaya@erzincan.edu.tr \\ ${ }^{1}$ Erzincan Binali Yıldırım Üniversitesi, Mühendislik Fakültesi, Bilgisayar Mühendisliği Bölümü, Erzincan, Türkiye (ORCID: 0000-0003-2017-799X), \\ baran@erzincan.edu.tr \\ ${ }^{2}$ Fırat Üniversitesi, Teknoloji Fakültesi, Elektrik-Elektronik Mühendisliği Bölümü, Elazığ, Türkiye (ORCID: 0000-0002-7435-0906), stuncer@ firat.edu.tr
}

(İlk Geliş Tarihi Aralık 2020 ve Kabul Tarihi Ocak 2021)

(DOI: 10.31590/ejosat.845467)

ATIF/REFERENCE: Kaya, V., Baran, A. \& Tuncer, S. (2021). Dinamit Destekli Terör Faaliyetlerinin Önlenmesi İçin Derin Öğrenme Temelli Güvenlik Destek Sistemi. Avrupa Bilim ve Teknoloji Dergisi, (22), 81-85.

\section{Öz}

Günümüz toplumunda, insanları tehdit eden en önemli etmenlerden birisi terörizmdir. Terörizm bir toplumda, insanların düzen durumlarını bozarak, yaşam kalitesini etkilemektedir. Devletler ise terörle mücadele etmek için sürekli farklı yöntemler geliştirmektedir. $\mathrm{Bu}$ yöntemlerden birisi de terörle mücadele için makine öğrenmesinin bir alt alanı olan derin öğrenmenin kullanılmasıdır. Derin öğrenme, makine öğrenmesi alanında son yıllarda oldukça popülerlik kazanmıştır. Bu çalışmada, terör faaliyetlerini fark etmek ve önlemek için derin ögrenmeye dayalı VGG-16 mimarisi temel alınarak yeni bir model önerilmektedir. Önerilen model ile güvenlik kontrollerinde kullanılan kamera görüntülerinden alınan görüntülerde, insan ya da tren rayları üzerinde dinamit tespit edildiğinde, durumu hızla belirlemek ve uygun önlemleri almak için güvenlik görevlilerini uyaran bir sistem gerçekleştirilmiştir. Çalışmada kullanılan veri seti, internet ortamından indirilen dinamit resimleri düzenlenerek oluşturulmuştur. Önerilen modelin performansını değerlendirmek için, insan ya da tren rayları üzerinde bulunan dinamit resimleri test edilerek, \%98,4'lük başarı doğruluğu ve 0,024 kayıp oranıyla dinamit görüntüleri tespit edilmektedir.

\section{Deep Learning Based Security Support System For The Prevention Of Dynamite-Backed Terrorist Activities}

\begin{abstract}
In today's society, one of the most important factors that threaten people is terrorism. In a society, terrorism affects the quality of life by disrupting the order of people. On the other hand, states are constantly developing different methods to fight terrorism. One of these methods is the use of deep learning, a subfield of machine learning, to fight terrorism. Deep learning has gained considerable popularity in the field of machine learning in recent years. In this study, a new model was proposed the basically VGG-16 architecture based on deep learning to recognize and prevent terrorist activities. With the proposed model, when dynamite was detected on human or train tracks in the images taken from the camera images used in security controls, a system that alerts the security guards in order to quickly identify the situation and take the appropriate measures. The data set used in the study was created by editing images of dynamite downloaded from the internet environment. In order to evaluate the performance of the proposed model, dynamite image s found on human or train tracks were tested and dynamite images were determined with a success accuracy of $98.4 \%$ and a loss rate of 0.024 .
\end{abstract}

Keywords: Dynamite detection, Deep learning, Convolutional neural network

\footnotetext{
${ }^{*}$ Sorumlu Yazar: Erzincan Binali Yıldırım Üniversitesi, Mühendislik Fakültesi, Bilgisayar Mühendisliği Bölümü, Erzincan, Türkiye, ORCID: 00000001-6940-3260, vkaya@erzincan.edu.tr
} 


\section{Giriş}

Suç oranlarının giderek artığı günümüz şartlarında, güvenlik için alınan tedbirler önemli bir ihtiyaç haline gelmektedir. Günümüz toplumuna yönelik tehditlerin başında terör olayları gelmektedir. Terör olaylarının insanlar için önemli bir tehdit olduğu bilinmekte ve engellemek için çeşitli çabalar sarf edilmektedir[1-3]. Bu sorunu çözmek için terör olaylarını anlamak veya nasıl gerçekleştirileceğini tahmin etmek üzerine [4-5] çeşitli disiplinlerde çalışılmaktadır [6-9]. Bilişim alanında ise son yıllarda terör olaylarını engellemek için makine öğrenmesi algoritmaları kullanılmaktadır [10-12]. Bu kapsamda özellikle makine öğrenmesinin bir alt alanı olan derin öğrenme yöntemleri kullanılarak büyük ilerlemeler sağlanmıştır [13]. Derin öğrenme, büyük miktarda etiketli veri kullanarak nesne algılama ve sınıflandırma alanlarında popülerlik kazanmıştır $[13,14]$. Böylece derin öğrenme modelleri, bir terör eyleminin olup olmayacağını ve terörist faaliyetlerine yol açan farklı unsurların tahminlerini yapmak için de kullanılabilmektedir. Bununla birlikte belirli bir terör olayının meydana geleceğini tahmin etmek zor bir görevdir [15]. Terörle mücadele için bu tahminlerin anlaşılması oldukça önemlidir. Derin öğrenme, bu tahminleri verimli bir şekilde yapabilmekte ve bireylerin hayatlarını korumak için yeni yöntemlerin geliştirilmesine yardımcı olmaktadır. Bu yöntemler yardımıyla, bir terör eylemi fiilen gerçekleşmeden önce güvenlik güçlerinin terör olayları ile başa çıkmasını sağlanabilmektedir [16].

$\mathrm{Bu}$ çalışmada, derin öğrenme yöntemleri kullanılarak, dinamit destekli terör faaliyetlerini engelleyebilmek ve güvenlik güçlerinin patlama gerçekleşmeden önce önlem almasına yardımcı olmak için yeni bir model önerilmiştir. Önerilen model için hazır veri seti olmadığından, internet üzerinden dinamit resimleri indirilerek yeni bir veri seti oluşturulmuştur. Bu model insan ya da tren yolu üzerinde dinamit tespit eden ve tanıyan yeni bir yaklaşım sunmaktadır.

Çalışmanın geri kalan kısmı aşağıdaki şekilde düzenlenmiştir: Alandaki son araştırma çalışmalarını vurgulamak için konu ile ilgili çalışmalar bölüm 2'de sunulmuştur. Önerilen materyal ve yöntemler bölüm 3 'te açıklanmaktadır. Son olarak, sonuçlar ve öneriler bölüm 4'te verilmektedir.

\section{2. İlgili Çalışmalar}

Derin sinir ağlarını kullanarak terör faaliyetlerinin tahminini yapmaya çalışan bir çalışmada, terör faaliyetinin davranışını anlamak için saldırının türünün intihar olup olmadığı, saldırının başarılı olup olmadığı, ne tür bir silah kullanılabileceği, hangi bölgenin hedef alınabileceği ve ne tür bir teröristin kullanılacağına dayalı beş farklı faktör belirlenmektedir [16]. Çalışmada tek katmalı sinir ağı ve derin sinir ağı tabanlı modeller geliştirilerek, derin sinir ağına dayalı modelin başarı oranının daha yüksek olduğu görülmüştür [16].

Terör olaylarını tahmin etmek için gerçek zamanlı büyük veri analizi içeren bir çalışmada [17], gerçek zamanlı terör olay verileri kullanılarak, terör risk seviyelerini hesaplamak ve tahmin etmek için yeni bir model önerilmektedir. Önerilen modelin risk değerlerinin önceden tanımlanan risk aralığ 1 içinde olduğu görülmektedir. Başka bir çalışmada ise, terörist saldırılarını tahmin etmek için saldırı türü, saldırı bölgesi ve saldırı türünü sinıflandıran silah türü tahminleri olmak üzere üç öngörüsel saldırı modeli sunulmaktadır. Bu modeller, destek vektör makinesi (SVM), Yapay Sinir ağı (YSA), Naïve Bayes (NB), Random Forest (RF), REP Ağacı ve J48 gibi makine öğrenme algoritmaları kullanılmış ve bulgulara göre, RF'nin diğerlerine kıyasla daha iyi performans gösterdiği ortaya konulmuştur [18].

Evrişimli sinir ağının derin öğrenme ile tehlikeli nesne tespitine yönelik bir çalışmada, tehlikeli madde tespiti için derin öğrenme temelli evrişimli sinir ağına sahip bir algoritma önerilmiştir. Önerilen algoritmada nesne tespiti için evrişimli bir sinir ağı, pozitif ve negatif örneklerle eğitilerek, tehlikeli nesne olup olmadığını belirlemek için farklı ölçeklerde sürgülü pencere kullanılmaktadır [19].

Evrişim sinir ağ1 kullanılarak X-1şını görüntülerinden tehlikeli nesnelerin tespiti yapan bir çalışmada, X-1şını görüntü veri seti kullanılarak makas bıçak ve şişe tespit edilmektedir [20]. Başka bir çalışmada ise, benzin istasyonların da tehlikeli davranışları tespit etmek için derin öğrenme teknolojisine dayalı nesne tespit sistemi gerçekleştirilmiştir. Sistem, görüntüdeki tehlikeli nesneleri (sigara vb.) doğru ve hılı bir şekilde tespit edilebildiği kanıtlanmıştır [21].

Tehlikeli nesne algılama için özellik çıkarma ve sınıflandırma algoritmalarının bir analizi yapılan bir çalışmada ise, bir görüntüde bıçak gibi tehlikeli nesneleri doğru bir şekilde tanımlamak için bag of words, HOG-SVM, CNN ve önceden eğitilmiş Alexnet algoritmaları kullanılmıştır. Çalışmada, SVM ile birlikte önceden eğitilmiş Alexnet kullanılarak en iyi sonuç alındığı görülmüştür [22].

\section{Materyal ve Yöntem}

\subsection{Sistem Yapılandırması}

Derin öğrenme modeli, ağırlıklı olarak işlem birleşik cihaz mimarisi (Compute Unified Device Architecture (CUDA)) çekirdeği etkinleştirilmiş Grafik İşleme Birimine (GPU) dayanmaktadır. Çalışma, CUDA etkinleştirilmiş (896 CUDA çekirdeği), 4GB GPU GTX 1650 ve 8 GB Rasgele Erişim Belleği (RAM) ile gerçekleştirilmiştir. Ayrıca çalışma için önerilen model Python programlama dili kullanılarak yazılmıştır.

\subsection{Veri Seti}

Geliştirilin model için internet ortamından, 491 adet dinamit resmi ve 150 adet insan resimlerine ait el ve yüz görüntüleri indirilerek veri seti haline getirilmiştir [23, 24]. Veri seti içerisinde bulunun tüm resimler üç kanallı Kırmızı Yeşil Mavi (Red Green Blue (RGB)) formatında 48x48 piksel boyuna getirildikten sonra dinamit ve dinamit olmayan olarak etiketlenmiştir. Veri setine ait örnek resimler Şekil 1'de verilmiştir.

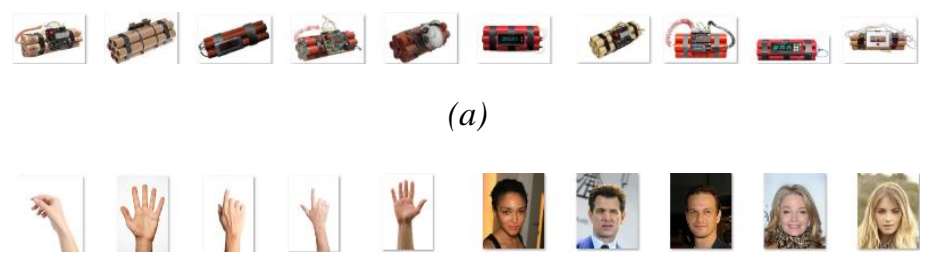

(b)

Şekil 1. Örnek veri seti (a) Dinamit (b) Dinamit olmayan. 


\subsection{Evrişimsel Sinir Ă̆ı Modeli}

Çalışmada insan ya da tren rayları üzerinde dinamit tespit etmek için Şekil 2' de sunulmuş yeni bir evrişimsel sinir ağ modeli önerilmektedir. Önerilen model için Tablo 1'de gösterilen katman türleri kullanılarak, 324.162 parametre elde edilmiştir.

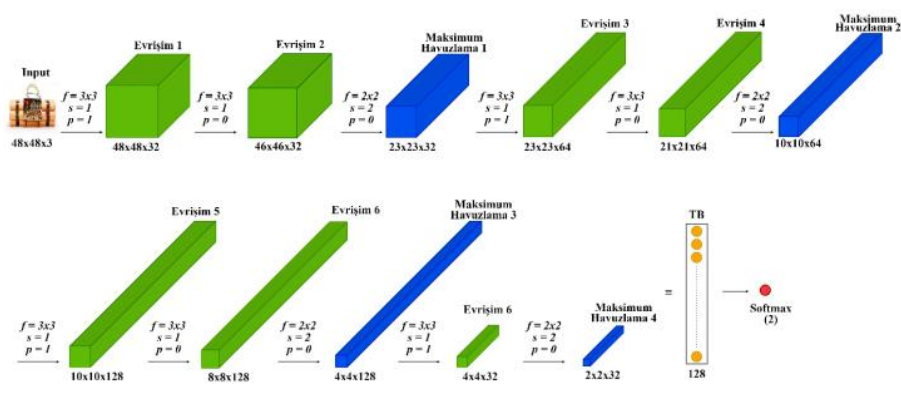

Şekil 2. Evrişimsel sinir ă̆ modeli

(f: filtre boyutu, s: adım, $p$ : dolgu, TB: tam bağll)

Tablo 1. Model katman türleri ve sayıları

\begin{tabular}{|l|c|}
\hline \multicolumn{1}{|c|}{ Katman Türü } & Katman Sayısı \\
\hline Evrişim & 7 \\
\hline Havuzlama & 4 \\
\hline Unutma & 4 \\
\hline Tam bağlı & 1 \\
\hline Sınıflandırma & 1 \\
\hline ReLU & 7 \\
\hline Toplam & $\mathbf{2 4}$ \\
\hline
\end{tabular}

Şekil 2'de verilmiş modelin evrişim katmanlarında Doğrultulmuş Doğrusal Birim (Rectified Linear Unit (ReLU)) aktivasyon fonksiyonu ve modelin eğitimi sırasında ağın aşırı öğrenmesini (ezberlemesini) engellemek için havuzlama katmanlarından sonra \% 25'lik unutma katmanı kullanılmıştır. Ayrıca modelde Adamax optimizasyon algoritması ve 50 eğitim tur sayısı (Epoch) kullanılmaktadır.

\section{Deneysel Sonuçlar}

Çalışmada önerilen model açık kaynaklı bir makine öğrenimi çerçevesi sunan Tensorflow aracılığıla, Python programlama dili kullanılarak yazılmış ve geliştirilmiştir. Çalışmada kullanılan veri seti, Tablo 2' de görülebileceği gibi eğitim, test ve doğrulama olmak üzere üç veri kümesine ayrılmıştır. Modelin eğitim sırasında eğitim ve test veri kümesi, test sırasında ise doğrulama veri kümesi kullanılmıştır.

Tablo 2. Veri kümesi

\begin{tabular}{|c|c|c|c|c|}
\hline \multirow{2}{*}{ Veri Seti } & $\begin{array}{c}\text { Eğitim } \\
(\mathbf{\% 6 0 )}\end{array}$ & $\begin{array}{c}\text { Test } \\
(\mathbf{\% 2 0})\end{array}$ & $\begin{array}{c}\text { Doğrulama } \\
(\mathbf{\% 2 0})\end{array}$ & Toplam \\
\cline { 2 - 5 } & 384 & 129 & 128 & 641 \\
\hline
\end{tabular}

Modelin eğitim işlemi sonucunda ölçülen precision (kesinlik), recall (duyarlılık), fl-skoru ve accuracy (doğruluk) performans ölçütlerinin matematiksel ifadeleri denklem (1-4)'de, performans sonuçları Tablo 3'de ve modelin doğruluk ve kayıp grafiği Şekil 3'de görülmektedir.

e-ISSN: 2148-2683

$$
\begin{aligned}
& \text { Precision }(\text { Kesinlik })=\frac{T P}{T P+F P} \\
& \text { Recall }(\text { Duyarlıllk })=\frac{T P}{T P+F N} \\
& f 1-\text { skoru }=2 \times \frac{\text { Kesinlik } \times \text { Duyarlllık }}{\text { Kesinlik }+ \text { Duyarlıllk }}
\end{aligned}
$$$$
\operatorname{Accuracy}(\text { Doğruluk })=\frac{T P+T N}{T P+F P+F N+T N}
$$

Tablo 3. Modelin performans sonuçları

\begin{tabular}{|l|c|c|c|}
\hline & Kesinlik & Duyarlılık & F1-skoru \\
\hline Dinamit & 0.99 & 0.99 & 0.99 \\
\hline İnsan & 0.96 & 0.96 & 0.96 \\
\hline \multicolumn{4}{|l}{} \\
\hline Doğruluk & & & 0.98 \\
\hline Makro ortalama & 0.98 & 0.98 & 0.98 \\
\hline Ağırlıklı ortalama & 0.98 & 0.98 & 0.98 \\
\hline
\end{tabular}
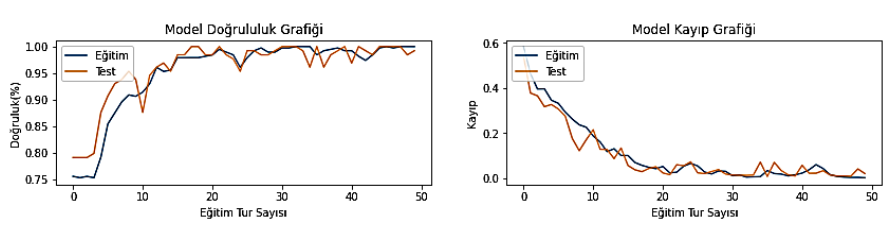

Şekil 3. Modelin doğruluk ve kayıp grafiğ $i$

Modelin doğruluğunu test etmek için ağın eğitim sırasında görmediği doğrulama veri seti kullanılarak test edilmiş ve $\% 98,4$ başarı doğruluğu ile 0,024 kayıp oranı elde edilmiştir. Ayrıca doğrulama veri kümesi kullanılarak Şekil 4'de gösterilen karışıklık matrisi elde edilmiştir.

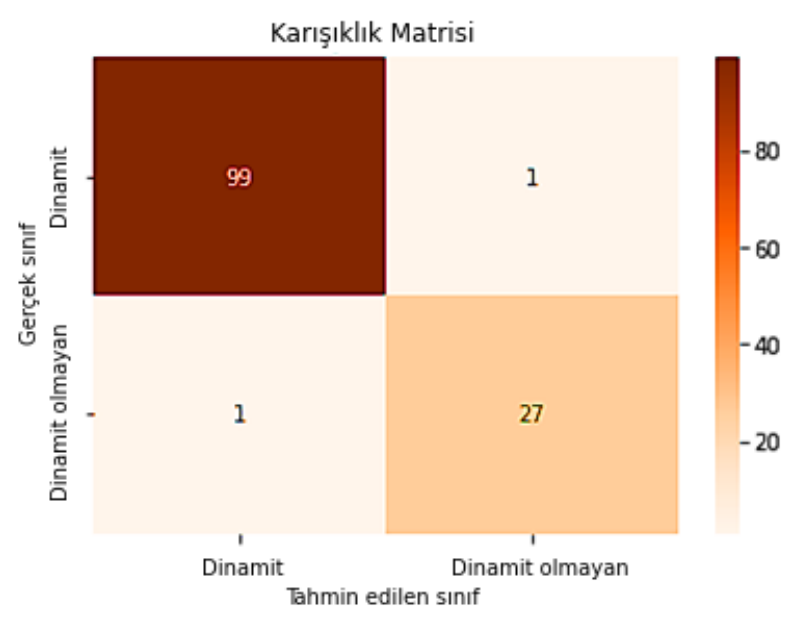

Şekil 4. Karışıklık matrisi

Tablo 3, Şekil 3 ve Şekil 4'de verilen sonuçlara göre modelin başarılı bir şekilde sınıflandırma yaptığı görülmektedir. Ayrıca modelin performans sonuçları, insan ya da tren rayları üzerinde bulunan dinamit görüntüleri kullanılarak test edilmiş ve Şekil 5'de sunulmuştur. Elde edilen test sonuçlarına göre önerilen modelin üstün bir başarı gösterdiği görülmektedir. 

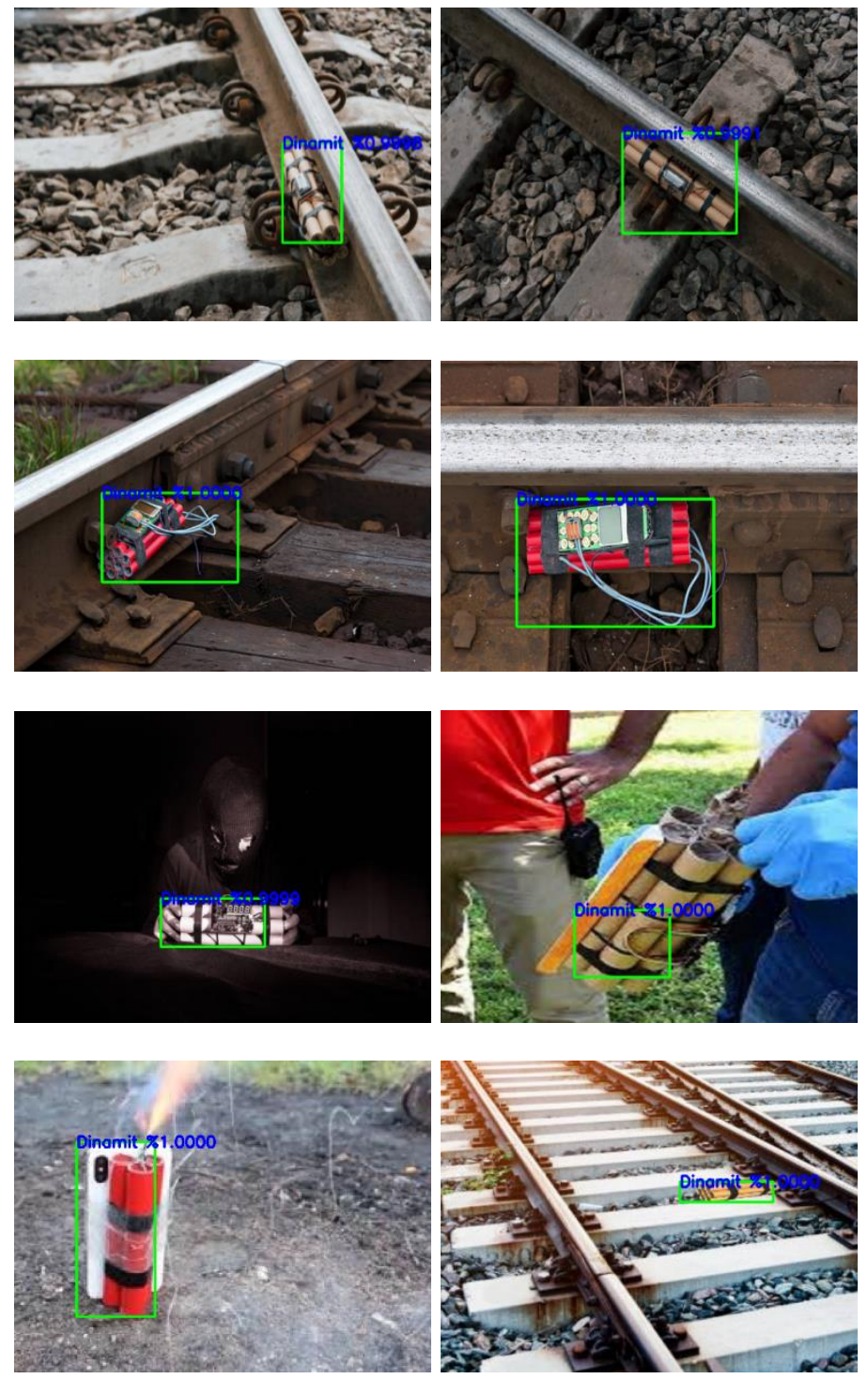

Şekil 5. Farklı görüntülere sahip örnek test sonuçları

\section{Sonuçlar ve Öneriler}

Bu çalışmada, derin öğrenme mimarilerinden evrişimsel sinir ağ kullanılarak, dinamit destekli terörist faaliyetleri engellemeye çalışmak ve güvenlik güçlerinin bir olay gerçekleşmeden önce önlemesine yardımcı olmak için dinamit tespit sistemi önerilmiştir. Önerilen model için hazır veri seti olmadığından, internet ortamından dinamit resimleri indirilerek yeni bir veri seti oluşturulmuştur. Geliştirilen model eğitim ve test veri kümesi ile eğitildikten sonra ağın hiç görmediği doğrulama veri kümesi ile test edilerek \%98,4 oranında başarı elde edilmiştir. Önerilen model insan ya da tren rayları üzerinde bulunun dinamit görüntüleri ile test edildiğinde, üstün bir performans sergilemiştir. Çalışmanın doğrudan güvenlik alanlarında uygulanabilir olması çalışmanın özgün değerini artırarak, gelecekteki çalışmalara katkı sağlayacağı beklenilmektedir.

\section{Kaynakça}

1. Yuan, J., \& Guo, C. (2018, June). A deep learning method for detection of dangerous equipment. In 2018 Eighth International Conference on Information Science and Technology (ICIST) (pp. 159-164). IEEE.

2. Ionescu, B., Ghenescu, M., Răstoceanu, F., Roman, R., \& Buric, M. (2020). Artificial Intelligence Fights Crime and Terrorism at a New Level. IEEE MultiMedia, 27(2), 55-61.

3. Makarenko, T. (2004). The crime-terror continuum: tracing the interplay between transnational organised crime and terrorism. Global crime, 6(1), 129-145.

4. Krieger, T., \& Meierrieks, D. (2011). What causes terrorism?. Public Choice, 147(1-2), 3-27.

5. Ouassini, N., \& Verma, A. (2018). Socio-economic inequality or demographic conditions: a micro-level analysis of terrorism in Jharkhand. Journal of Victimology and Victim Justice, 1(1), 63-84.

6. Mnih, V., Kavukcuoglu, K., Silver, D., Rusu, A. A., Veness, J., Bellemare, M. G., ... \& Petersen, S. (2015). Human-level control through deep reinforcement learning. nature, 518(7540), 529-533.

7. Lake, B. M., Salakhutdinov, R., \& Tenenbaum, J. B. (2015). Human-level concept learning through probabilistic program induction. Science, 350(6266), 1332-1338.

8. Karatas, G., Demir, O., \& Sahingoz, O. K. (2018, December). Deep learning in intrusion detection systems. In 2018 International Congress on Big Data, Deep Learning and Fighting Cyber Terrorism (IBIGDELFT) (pp. 113-116). IEEE.

9. Ilgin, F. Y. (2020). Energy-based spectrum sensing with copulas for cognitive radios. Bulletin of the Polish Academy of Sciences. Technical Sciences, 68(4).

10. Alhamdani, R., Abdullah, M., \& Sattar, I. (2018). Recommender system for global terrorist database based on deep learning. International Journal of Machine Learning and Computing, 8(6).

11. Saeed, Y., Ahmed, K., Zareei, M., Zeb, A., Vargas-Rosales, C., \& Awan, K. M. (2019). In-vehicle cognitive route decision using fuzzy modeling and artificial neural network. IEEE Access, 7, 20262-20272.

12. Huamaní, E. L., Alicia, A. M., \& Roman-Gonzalez, A. (2020). Machine Learning Techniques to Visualize and Predict Terrorist Attacks Worldwide using the Global Terrorism Database. Machine Learning, 11(4).

13. Ali Shah, S. A., Uddin, I., Aziz, F., Ahmad, S., AlKhasawneh, M. A., \& Sharaf, M. (2020). An enhanced deep neural network for predicting workplace absenteeism. Complexity, 2020.

14. Sai, B. K., \& Sasikala, T. (2019, November). Object Detection and Count of Objects in Image using Tensor Flow Object Detection API. In 2019 International Conference on Smart Systems and Inventive Technology (ICSSIT) (pp. 542546). IEEE.

15. Bakker, R., Hill Jr, D. W., \& Moore, W. H. (2014). Modeling terror attacks: A cross-national, out-of-sample study. Understanding Terrorism (Contributions to Conflict Management, Peace Economics and Development, Volume 22) Emerald Group Publishing Limited, 22, 51-68.

16. Uddin, M. I., Zada, N., Aziz, F., Saeed, Y., Zeb, A., Ali Shah, S. A., ... \& Mahmoud, M. (2020). Prediction of Future Terrorist Activities Using Deep Neural Networks. Complexity, 2020. 
17. Toure, I., \& Gangopadhyay, A. (2016, May). Real time big data analytics for predicting terrorist incidents. In 2016 IEEE Symposium on Technologies for Homeland Security (HST) (pp. 1-6). IEEE.

18. Verma, C., Malhotra, S., \& Verma, V. (2018). Predictive modeling of terrorist attacks using machine learning. International Journal of Pure and Applied Mathematics, 119, 06.

19. Yang, S., Sun, J., Duan, Y., Li, X., \& Zhang, B. (2020, January). Dangerous object detection by deep learning of convolutional neural network. In Second Target Recognition and Artificial Intelligence Summit Forum (Vol. 11427, p. 1142722). International Society for Optics and Photonics.

20. Zou, L., Yusuke, T., \& Hitoshi, I. (2018, December). Dangerous objects detection of X-ray images using convolution neural network. In International Conference on Security with Intelligent Computing and Big-data Services (pp. 714-728). Springer, Cham.

21. Chang, Y., Du, Z., \& Sun, J. (2019, August). Dangerous behaviors detection based on deep learning. In Proceedings of the 2nd International Conference on Artificial Intelligence and Pattern Recognition (pp. 24-27).

22. Kibria, S. B., \& Hasan, M. S. (2017, December). An analysis of feature extraction and classification algorithms for dangerous object detection. In 2017 2nd International Conference on Electrical \& Electronic Engineering (ICEEE) (pp. 1-4). IEEE.

23. Istock, https://www.istockphoto.com, Erişim Tarihi Eylül, 20, 2020.

24. Liu, Z., Luo, P., Wang, X., \& Tang, X. (2015). Deep learning face attributes in the wild. In Proceedings of the IEEE international conference on computer vision (pp. 37303738). 